\title{
Acute Placental Insufficiency Two Weeks After an Asymptomatic COVID-19 Maternal Infection: The Deleterious Effects of The SARS-Cov-2 Placentitis Associated with B.1.617.2 (Delta) Variant. Report of Two Cases.
}

Loredana Delle Chiaie ( $\sim$ loredana.delle-chiaie@web.de)

Klinikum Stuttgart https://orcid.org/0000-0002-4457-0297

llona Stolpner

Katrin Baltz-Ghahremanpour

Research Article

Keywords: acute placental insufficiency, SARS-CoV-2 Infection, Placentitis, maternal

Posted Date: March 7th, 2022

DOI: https://doi.org/10.21203/rs.3.rs-1343858/v1

License: (c) (i) This work is licensed under a Creative Commons Attribution 4.0 International License.

Read Full License 


\section{Abstract}

We present two cases of acute severe placental insufficiency in the late second trimester and in the third trimester of pregnancy, emerging within 14 days following a mild COVID-19 (Delta variant) disease in two not vaccinated healthy women with hitherto unremarkable pregnancies. The pathognomonic histopathological findings and the RT-PCR positivity of the placenta suggest the SARS-CoV-2 placentitis as the possible cause of the severe fetal distress. At present, propagation of vaccination and a strict fetomaternal surveillance in the first weeks after maternal infection seem to be the only way to protect the gestation from the risks of acute placental insufficiency associated with the SARS-CoV- 2 infection.

\section{Introduction}

Two years after the outbreak of COVID-19 pandemic a lot of knowledge about the course of the disease, the viral pathogenicity, the therapies, and the prevention of the infection have been acquired. Nevertheless, we are still far away from the end of it.

The impact of the SARS-CoV-2 infection in pregnancy raises great concern about the possible risks for the mothers and their unborn children.

First published data has shown somewhat contradictory findings about the consequence of the infection for pregnancy outcome, but recent reports indicated an unequivocal higher rate of preterm delivery, preeclampsia and maternal mortality in SARS-CoV-2 infected mothers. On the other hand, the risk of vertical transmission is very low (0.3-3.2\%), teratogenic effects have not been observed and the infected newborns mostly have a mild course of disease so that there is only little direct impact of the maternal infection on the unborn child [1-7].

In the last months, we observed an evolution of the pandemic not only due to the development of the new SARS-CoV-2 variants. The lack of data about the Covid-19-vaccination of pregnant women, and hence the vaccine hesitancy before and during pregnancy has left a big part of childbearing women unprotected from the infection. Thus, the number of infected pregnant women exploded.

The B.1.617.2 (delta) variant is circulating in Germany since March 2021 and from June 2021 till January 2021 has been the dominant one.

The CDC reported a 4-fold risk of abortion/stillbirth in infected mothers during the B.1.617.2 (delta) variant dominance, and the severely decreased placental function caused by SARS-CoV-2 placentitis seems to be a possible explanation [8-13].

Meanwhile pathognomonic histopathological patterns in cases of SARS-CoV-2 placental infection have been described (inflammatory intervillositis, increased perivillous fibrin, villous trophoblast necrosis): all these cases are associated with poor fetal outcome $[8,10-15]$. 
We present two cases of acute placental insufficiency and fetal distress emerging about 14 days after the diagnosis of maternal SARS-CoV-2 infection with paucisymptomatic clinical course during the dominance of the B.1.617.2 (delta) variant of the virus.

\section{Case 1}

involved a 34-year-old healthy pregnant woman $(2 \mathrm{G}, \mathrm{OP})$ at 23 weeks 6 days of gestation, who presented to our observation at the first day after her 14-day isolation due to a mild SARS-CoV-2 infection (B.1.617.2). Until that moment, the pregnancy had been uneventful. The fetal scan showed a FGR (fetal Growth Restriction) with pathological maternal and fetal arterial flow (AREDF), but still positive pulsatiliy of the DV (Ductus Venosus). No other risks for placental insufficiency were known, the NIPT (Not Invasive Prenatal Test) showed a low risk for Trisomy 21, 18 and 13, CMV and Toxoplasmosis serology were uneventful.

The patient was normotensive, asymptomatic and with normal blood values without any indication for HELLP-Syndrome. The sFIt/PLGF quotient showed a high risk for a preeclampsia. The parents were counselled about the very poor fetal outcome in case of delivery at that moment $(23 w+6 d)$, so that they preferred an expectant management, being aware of the high risk for IUD (Intrauterine fetal Death). We induced the fetal lung maturation (betamethasone $12 \mathrm{mg} I \mathrm{M}$ for two doses 24 hours apart), monitored the maternal arterial pressure and well-being without fetal monitoring. At 25 weeks of gestation, the earliest point at which delivery could be recommended, we started the fetal surveillance and found a deterioration of the DV velocimetry (AREDF) and a pathologic CTG tracing (FIGO: silent with decelerations). The patient was still fine, without clinical and laboratory signs of preeclampsia. After a repeated counselling by the neonatologists, she consented the urgent cesarean section in spite of the high risk of hypoxic-ischemic encephalopathy in the newborn. The patient delivered a $450 \mathrm{~g}$ boy, APGAR score $2 / 4 / 7$, umbilical artery $\mathrm{pH}$ 6.99. The initial neonatal course was complicated by respiratory distress syndrome and other complications consistent with the prematurity, the extremely low birth weight and the hypoxia-acidosis at delivery. The infant recovered slowly and could be dismissed after 3 months in a relatively good clinical condition appropriate to his extreme prematurity.

Macroscopically, the placenta was eutrophic, but diffusely affected by large irregular, solid, and whitish areas (Fig. 1). Microscopically, prominent villous trophoblastic necrosis $(>60 \%)$ and increased perivillous fibrin deposition could be observed. A positive RT-PCR (high viral load) was obtained from the fetal side of the placenta, although the neonatal nasopharyngeal swab was negative and the maternal one weakly positive (low viral load), suggesting a viral infection of the placenta (SARS-CoV-2 Placentitis).

\section{Case 2}

a 31-year old healthy pregnant woman (1G,0P) at 35 weeks +1 day of gestation was referred to our clinic because of a non-reassuring fetal heart rate tracing 14 days after her positive nasopharyngeal swab RTPCR result for SARS-CoV-2, at just the first day after her isolation. She reported an asymptomatic course of the COVID-19 disease, but she noticed a reduction of the fetal movements in the last few days. Being 
the B.1.617.2 (delta) variant dominant in this period, there was no sequencing of the SARS-CoV-2 genome. After few minutes of FHR-registration (fetal heart rate) we suspected a fetal acidosis. A quick ultrasound scan showed a hypotrophic fetus with a pathological Doppler flow of the umbilical artery, a cerebral vasodilatation, a high pulsatile flow in the DV and a reduced amount of amniotic fluid. Thus, we confirmed the suspicion of fetal acidosis and indicated an emergent cesarean section. We delivered a depressed, acidotic, hypotrophic girl (1800g, APGAR score 1/5/7, umbilical artery pH 7.06). After an uncomplicated first care she was transferred to the intermediate NICU for monitoring. She recovered quickly and could be dismissed after a few days.

Macroscopically, the placenta was mild hypotrophic ( $10^{\circ}$ percentile) and inhomogeneously affected by large irregular, solid and whitish areas. Microscopically, prominent villous trophoblastic necrosis (40-45\%) and increased intervillous inflammatory infiltration (villitis and intervillositis) could be observed (Fig. 2). A positive RT-PCR (high viral load) was obtained from the fetal side of the placenta even though the neonatal and maternal nasopharyngeal swab were negative, as well as in case 1 , suggestive of a viral infection of the placenta (SARS-CoV-2 Placentitis).

\section{Discussion}

We present two cases of acute severe placental insufficiency in the late second trimester and third trimester of pregnancy, emerging within 14 days following a mild COVID-19 (Delta variant) disease in two not vaccinated healthy women with hitherto unremarkable pregnancies. The pathognomonic histopathological findings and the RT-PCR positivity of the placenta suggest the SARS-CoV-2 placentitis as the possible cause of the severe fetal distress.

Since the beginning of the pandemic, we did not observe any similar cases with previous SARS-CoV-2 virus variants at our perinatal centre. Therefore, we suppose that the Delta variant could be more aggressive on the placenta, causing severe placental damage and insufficiency, as reported in preceding publications $[8,9]$.

We did not find any correlation between the severity of the maternal disease and the severity of the placental malfunction, which manifested two weeks after SARS-CoV-2 infection. For this reason, we recommend an intensive feto-maternal surveillance especially in the first weeks following the maternal infection, independently of the severity of the disease.

Different SARS-CoV-2 variants may have different pathogenicity on the placenta, as observed in pregnancies infected with the Wild Type and Delta variant $[1,8]$ At the present time, the Omicron variant (B.1.1.529) surpasses the other variants, thus becoming the dominant one in Germany, as well as in many other countries. The effects of Omicron and other possible new variants on pregnancy outcome are not yet known and should be observed in the next months. It is eligible that the growing number of immunized women of childbearing age (vaccinated or recovered from infection) will moderate the clinical course of the disease on the mother and of the placental impairment. 
Further research is necessary to understand the effects of the new virus variants and of the increasing rate of immunization on pregnancy outcome. At present, propagation of vaccination and a strict fetomaternal surveillance in first weeks after maternal infection seem to be the only way to protect the gestation from the risks of acute placental insufficiency associated with the SARS-CoV- 2 infection.

\section{Declarations}

\section{Author contributions}

L. Delle Chiaie: conception and design of the study, acquisition of data and data analysis, manuscript writing and editing. I.Stolpner: acquisition of data, manuscript writing and revision. K. BaltzGhahremanpour: acquisition of the data.

Conflict of interest: The authors declare that they have no conflict of interest.

Funding: None

Ethical approval: This study used exclusively data from an existing database of the Hospital (perinatology tertiary centre Olgahospital/Frauenklinik, Stuttgart). An institutional review board approval was not required.

Informed consent: We used only clinical data and tissue images, no identifiable personal data was gathered and published so that an informed consent is not necessary.

\section{References}

1. Shanes ED, Mithal LB, Oter S, Azad H, Miller E, Goldstein JA: Placental Pathology in COVID-19. Am J Clin Pathol 2020; 154:23-32

2. Villar J, Ariff S, Guiner R et al. Maternal and neonatal morbidity and Mortality among pregnant women with and without COVID-19 Infection: The INTERCOVID Multinational Cohort Study. Jama Pediatr 2021; 175 (8):817-826

3. Papegeorghiu A, Deruelle P, Gunier R et al. Preeclampsia and COVID-19: results from the INTERCOVID prospective longitudinal study. Am J Obstet Gynecol 2021 Sep;225(3):289.e1-289.e17

4. Mullins E, Hudak ML, Banerjee J, Getzlaff T, Townson J, Barnette K, Playle R, PerryA, Bourne T, Lees CC: PAN-Covid investigators and National Peinatal COVID-19 Registry Study Group. Pregnancy and neonatal Outcomes of COVID-19: coreporting of common outcomes from PAN-COVID and AAPSONPM registries Ultrasound Obstet Gynecol 2021; 57:573-581

5. Ping Wong Y, Yee Khong T, Chin Tan G: The Effects of COVID-19 on Placenta and Pregnancy: what do we know so far? Diagnostics 2021,11,94

6. Di Mascio D, Buca D, Berghella V, Khalil A, Rizzo G, Odibo A, Saccone G, Galindo A, Liberati M, D'Antonio F: Counseling in maternal-fetale medicine: SARS-CoV-2 Infection in pregnancy. Ultarsound 
Obstet Gynecol 2021; 57: 687-697

7. Moltner S, de Vrijer B, Banner H. Placental inferction and intrauterine growth restriction following SARS-CoV-2 infection. Archives of Gyenecology and Obstetrics 2021; 304: 1621-1622

8. Shook L, Brigida S, Regan J, Flynn J, Mohammadi A, Etemad B, Siegel M,Clapp M, Li J, Roberts D, Edlow A: SARS-CoV-2 placentitis associated with B.1.6172.2 (Delta) variant and fetal distress or demise. J Infect Dis. 2022; Jan 13;jiac008.doi:10.1093/infdis/jiac008

9. De Sisto CL, Wallace B, Simeone RM, et al. Risk for Stillbirth among women with and without COVID19 at delivery hospitalization-Unites States, March 2020-September 2021. MMWR Morb Mortal Wkly Rep.2021; 70(47): 1640-1645

10. Garrido Pontnou M, Navarro A, Camacho J, Crispi F et al. Diffuse trophoblast damage is the hallmark of SARS-Cov-2-associated fetal demise. Mod Pathol 2021 Sep;(34)9: 1704-1709

11. Libbrecht S, Van Cleemput J, Vandekerckhove L et al. A rare but devastating cause of twin loss in near term pregnancy highlighting the features of severe SARS-CoV-2 placentitis. Histopathology 2021 Oct;79 (4):674-676

12. Lineham L,O` Donoghue K, DineenS, White J, Higgins JR: SARS-CoV-2 placentitis: an uncommon complication of maternal COVID-19. Placenta 2021; 014:261-266

13. Schwartz D, Baldewijns M, Benachi, A, Bugatti A et al. Chronic histiocytic Intervillositis with Trophoblast Necrosis is a risk factor associated with placental infection from Coronavirus Disease (COVID-19) and Intrauterine Maternal-Fetal Severe Acute Respiratory Syndrome Coronavirus 2 (SATSCoV-2) Transmission in live-born and stillborn infants. Arch Pathol Lab Med 2021; 145:517-526

14. Torous VF, Watkins JC, Roberts DJ: Response to „diffuse trophoblast damage is the hallmark of SARS-CoV-2-associated fetal demise “. Mod Pathol 2021; Nov 29; 1 -2

15. Watkins JC, Torous VT, Roberts DJ: Defining severe acute respiratory Syndrome Coronavirus 2 (SARS-CoV-2) Placentitis. Arch Pathol Lab Med 2021 Nov 1; 145 (11): 1341-1349

\section{Figures}

\section{Figure 1}

Maternal and fetal side of the Placenta (Case 1): eutrophic but diffusely affected by large irregular, solid, and whitish areas (widespread villous trophoblastic necrosis )

\section{Figure 2}

Histology (microscopic) of the placenta from Case 2: A) H/E-appearance shows the triad of perivillous fibrin depositation, mixed inflammatory intervillositis and villous trophoblast necrosis, characteristic for 
SARS-CoV-2 placentitis.

B) Immunohistochemistry for CD8-T-cells on the surface of syncytiotrophoblast . 